\title{
Early Complications in Proximal Femoral Nailing Done for Treatment of Subtrochanteric Fractures
}

\author{
B Kanthimathi, DNB Orth, VL Narayanan, MS Orth \\ Division Of Orthopaedics, Rajah Muthiah Medical College Annamalai University, Tamilnadu, India
}

\begin{abstract}
Aim:To analyse the early complications following the use of PFN in subtrochanteric fractures. Background: Osteosynthesis with PFN in subtrochanteric fracture features the advantages of high rotational stability of the head-neck fragment, an unreamed implantation technique and the possibility of static or dynamic distal locking. However, the use of the nail requires technical expertise and is accompanied by some risks of error which can lead to osteosynthesis failure. Methods: Between May 2009 and May 2011, 50 consecutive patients with PFN fixations for subtrochanteric fractures were observed for intraoperative and postoperative complications. Results: We identified intraoperative technical difficulties in four patients and six patients showed postoperative complications. Conclusion: When subtrochanteric fractures are to be stabilised with a PFN, the precise and expert technical performance of implantation is the basic surgical requirement. Good reduction with minimal dissection and the use of an appropriate implant is necessary to avoid treatment failure.
\end{abstract}

Key Words:

Subtrochanteric fractures, trochanteric fractures, proximal femoral nail, PFN.

\section{INTRODUCTION}

Subtrochanteric fractures account for 7 to $44 \%$ of all proximal femoral fractures ${ }^{1-3}$. The inherent instability of the fracture and the enormous muscle forces acting across the fracture fragments render treatment options difficult ${ }^{4}$. Often, these fractures are communited. A medial buttress is important to minimise implant stress and fatigue failure ${ }^{5-7}$, but when communition is severe, this cannot be achieved. Options for surgical stabilization of subtrochanteric fractures include dynamic hip screw, gamma nail, proximal femoral nail (PFNs) and proximal femoral plates. Intramedullary devices such as PFNs are biomechanically stronger and more rigid compared to extramedullary devices such as dynamic hip screws. Fixation with PFN using Association for Osteosynthesis/Association for Study of Internal Fixation (AO/ASIF) techniques has been reported to produce superior results compared to other fixation devices? Osteosynthesis with the PFN offers the advantages of high rotational stability of the head - neck fragment, an unreamed implantation technique and the possibility of static or dynamic distal locking ${ }^{10}$.

The operative technique of proximal femoral nailing for subtrochanteric fractures has a steep learning curve $\mathrm{e}^{11,12}$. Precise fracture reduction and adherence to the principles of PFN are crucial in the management of subtrochanteric fractures. We report our early experience with PFN in the management of subtrochanteric fractures and the accompanying complications.

\section{MATERIALS AND METHODS}

Fifty consecutive patients with subtrochanteric fractures who underwent intramedullary fixation with PFN, all performed by a single surgeon in our hospital during two-year period from May 2009 to May 2011 were included in this study. The Seinsheimer classification ${ }^{1}$ classification system was used to classify the fractures (Table I). All patients underwent femoral nailing.

All closed subtrochanteric fractures in adults treated with short PFNs with a minimum follow up period of one year were included in this study. Those with open subtrochanteric fractures and those treated with long PFNs were not included in this study. Postoperative rehabilitation protocol included: Day 0, static quadriceps exercises and ankle pump; Day 1, in bed, knee mobilisation exercises; Day 2, if fracture construct and fixation stable, partial weight bearing started as tolerated; if fracture construct and fixation unstable, weight bearing started after 6 weeks.

All patients who underwent surgery with short PFNs were placed in supine position on the fracture table. The limb was kept in traction and adducted. Entry point was visualisee under $\mathrm{C}$-arm guidance and a guide wire passed across the fracture site. Under C-arm guidance, a short PFN with proximal and distal locking was inserted to fix the fracture.

\section{RESULTS AND DISCUSSION}

In the present study, fifty patients were treated with PFN, of which ten encountered complications and treatment failure. 
Table I: SEINSHEIMER Classification

\begin{tabular}{|ll|}
\hline Type & Pattern of Fracture \\
\hline Type I & Nondisplaced or with less than $2 \mathrm{~mm}$ of displacement \\
Type II & Two part fracture \\
II a & Transverse \\
II b & Spiral configuration with lesser trochanter attached to proximal fragment. \\
II c & Spiral configuration with lesser trochanter attached to distal fragment. \\
Type III & Three part fractures. \\
III a & Three part spiral configuration with lesser trochanter a part of the third fragment. \\
III b & Three part spiral configuration with the third part a butterfly fragment. \\
Type IV & Comminuted with four or more fragments. \\
Type V & Subtrochantric - intertrochanteric configuration \\
\hline
\end{tabular}

Table II: Patient demographics and outcome

\begin{tabular}{|llc|}
\hline Parameters & Number \\
\hline Mean age (years) & Male & 46 \\
Gender & Female & 32 \\
& Right & 18 \\
Affected Limb & Left & 30 \\
& & 20 \\
Mean operating time (min) & Closed & 31.5 \\
Reduction technique & Open & 11 \\
& & 12.6 \\
Mean time for fracture union (weeks) & 20 \\
Complication (\%) & & \\
\hline
\end{tabular}

Table III: Complications of treatment

\begin{tabular}{|lccc|}
\hline Complications & $\begin{array}{c}\text { Number of } \\
\text { patients }\end{array}$ & $\begin{array}{c}\text { Percentage (\%) } \\
\text { (Seinsheimer classification) }\end{array}$ & $\begin{array}{c}\text { Fracture pattern } \\
\text { Implant broken }\end{array}$ \\
Proximal femur fracture & 2 & 4 & $\mathrm{II}$ \\
Screw cutout & 1 & 2 & $\mathrm{II}$ \\
Varus deformity & 1 & 2 & $\mathrm{II}$ \\
Inadequate fracture reduction & 1 & 2 & $\mathrm{II}$ \\
Improper positioning of distal locking screw & 1 & 2 & $\mathrm{II}$ \\
Abductor lurch & 1 & 2 & $\mathrm{III}$ \\
Superficial infection & 1 & 2 & $\mathrm{II}$ \\
Single neck screw & 1 & 2 & $\mathrm{IV}$ \\
\hline
\end{tabular}

Loss of reduction, inadequate fixation, a need to change implant and breakage of nails were considered as implant failure. The age distribution was from 24- to 60-years-old with an average age of 46-years-old. Thirty-two were males and eighteen were females. The right side was involved in thirty patients and the left side was involved in twenty patients. The mean follow up was twelve months (Table II).

Thirty-eight patients underwent closed reduction whereas twelve patients required open reduction. Ten out of fifty patients encountered complications. All were early complications and causes for failure were analysed. Complications include broken nail at the distal locking screw level(4\%), screw back out $(2 \%)$, perioperative proximal femur fracture $(2 \%)$, inadequate fracture reduction $(2 \%)$, varus deformity $(2 \%)$, improper positioning of distal locking screw $(2 \%)$, placement of single screw in the neck( $2 \%)$, abductor lurch (2\%) and superficial infection (2\%) (Table III).
Subtrochanteric fractures are generally associated with slightly higher failure rates compared to other fractures ${ }^{13}$. In a multicentre study, the failure rate of PFN secondary to poor reduction, malrotation or wrong choice of screw was reported to be $5 \%$, whereas the screw cut out rate was reported to be $0.6 \%{ }^{9}$. The reasons for failure include the higher intrinsic instability of the subtrochanteric fracture, poor control of fracture fragments ${ }^{13}$ and the difficulty in achieving the medial buttress due to communition. Intramedullary nails with an entry point in the piriformis fossa have a tendency to displace the proximal fragment into varus or may even explode the proximal femur during insertion ${ }^{14}$. The nail insertion point for the PFN should be at or slightly lateral to the tip of the greater trochanter ${ }^{14}$.

The proximal component of the PFN construct consists of two screws- the larger (lag) screw is designed to take the load, and the smaller screw (hip pin) provides rotational 


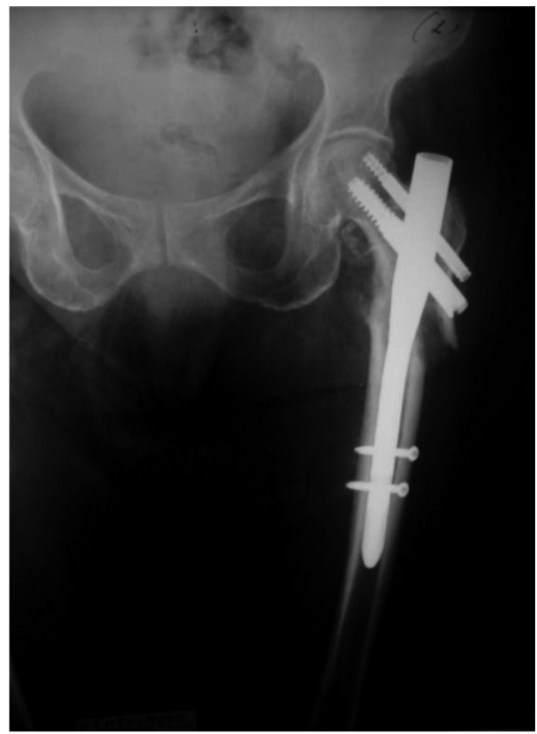

Fig. 1: Hip pin longer than lag screw.

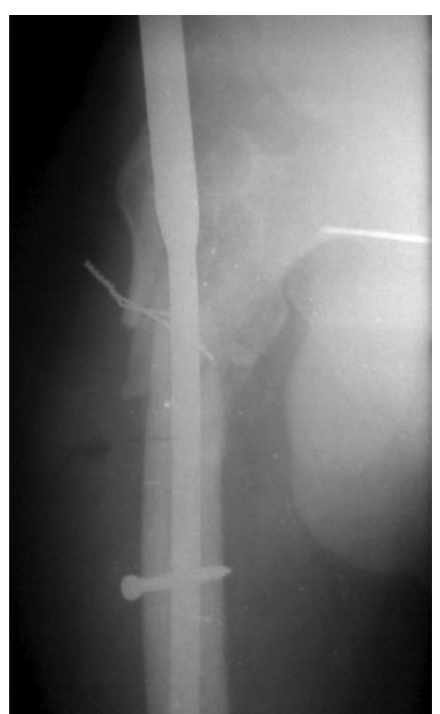

Fig. 3: Intraoperative femoral shaft fracture.

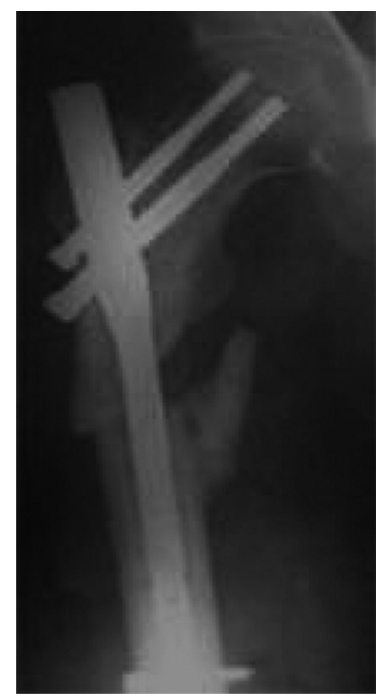

Fig. 5: Ten degree varus deformity of the femur.

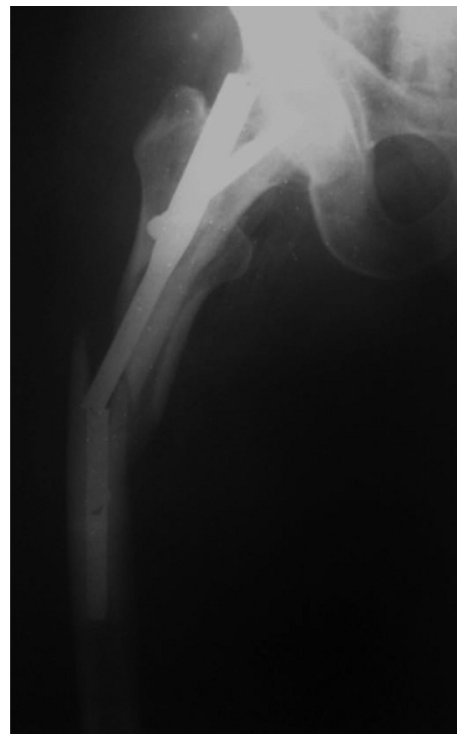

Fig. 2: Nail breakage at the distal interlocking level.

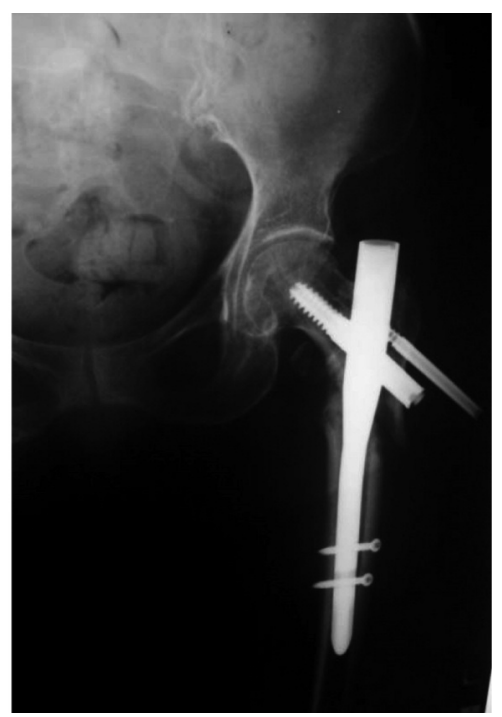

Fig. 4: Screw cut-out (hip pin backing out).

stability. The lag screw is longer than the hip pin, because if the hip pin was longer than the lag screw, vertical forces would increase on the hip pin thereby inducing cut out, a knife effect or Z- effect. This might force the hip pin to migrate into the joint and the lag screw to slide laterally ${ }^{15,16}$. The cut out rate with use of PFNs is reported to be $0.6 \%$ to $8 \%{ }^{17}$. Overall, ten patients $(20 \%)$ encountered complication. All complications were either perioperative, immediate postoperative or within three months of the index procedure. In one patient, the hip pin slide laterally at the sixth post operative week. This was due to incorrect choice of length for the lag screw and hip pin (the hip pin was longer than the lag screw, Figure 1).

Two complications consisted of implant failure at the distal locking screw hole with breakage of nail. One patient had breakage ten weeks postoperatively and the other at fourteen 
weeks (Figure 2). In both the patients, a short PFN was used. The distal tip of the nail was close to the fracture site $(3 \mathrm{~cm}$ and $4 \mathrm{~cm}$ ), an appropriate distance as cited in a previous publication ${ }^{11}$. We found no other clarification of the minimum effective distance between the distal locking screw and the fracture site. Ideally, the distal tip of the nail and the distal locking screw hole of the implant should be at a distance distal to the fracture that will provide sufficient mechanical stability but exert minimal internal mechanical stress on the implant. In subsequent cases, we used long PFNs for all comminuted fractures as they are inherently unstable even after fixation.

One patient suffered an intraoperative fracture of the proximal femur fragment. This complication was due to use of an incorrect entry point. For regular intramedullary nailing, the preferred entry point is at the piriformis fossa. but for PFNs it is at or slightly lateral to the tip of the greater trochanter ${ }^{14}$. If the PFN is passed at the piriformis fossa, the proximal fragment tends to displace into varus and may even explode the greater trochanter during nail insertion ${ }^{14}$. This is what happened with our patient (Figure 3). The nail was then exchanged and proximal femoral plating with bone grafting was performed. This patient had a delay in fracture healing (radiological union was not present until 20 weeks after surgery).

The hip pin should preferably be $10 \mathrm{~mm}$ shorter than the lag screw to reduce the load carried by the hip pin. One of our patients, who had a screw backing out (Figure 4), underwent a revision surgery and subsequently the fracture healed. In one patient, placement of both the lag screw and the hip pin in the neck was not possible. It was due to a very small femoral neck which could not accommodate both screws. Despite attempting to vary the position of the nail, both the screws could not be placed. It was decided to leave the patient with just the lag screw. This patient went on to union in the usual time frame. One patient had varus deformity due to failure of reduction at the fracture site (Figure 5). The varus was less than ten degrees and no attempt was made to revise. Fracture union was uneventful. One patient had wrong placement of distal locking screw. In this case short PFN was used. As the radiological union was evident at first review in six weeks no intervention was done. However the postoperative rehabilitation was not aggressive.The other two complications were superficial wound infection and abductor lurch when the patient started walking. The infection was treated with wound wash and specific antibiotics. The other patient with abductor lurch improved with progression of time.

In PFN fixations, proper alignment between the two main fragments and proper placement of the lag screws in the femoral head should be ensured ${ }^{18}$. It is imperative to reduce fractures with minimal dissection to achieve a stable fixation, with an emphasis on good closed reduction. Restoration of the axis and rotation between the head-neck fragment and the shaft is mandatory. A long nail is needed for fractures that extend distally. Multiple factors have been implicated as relevant to good outcomes; these include implant design, fracture stability, operative technique, surgeon skills and learning curve. Optimal reduction of the fracture, conformation of reduction in both anteroposterior and lateral views and accurate positioning of the nail and screws remain of crucial importance and should be obtained at all times to prevent the important complication of screw cut-out. Reduction in distal nail diameter, pre-reaming of the femoral canal one size bigger than the implant and meticulous placement of the distal locking screws without creating additional stress risers decreases the complication rate of femoral shaft fractures. Intramedullary fixation with $\mathrm{PFN}$ is contraindicated in patients with narrow femoral canals and/or abnormal curvature of the proximal femur.

\section{CONCLUSION}

Though the Asian version of the PFN is designed with a smaller diameter to accommodate for our population, it still poses problems in fixation. A surgeon needs to adhere to precise, meticulous and expert surgical technique. Placement of proximal locking screws - the lag screw and the hip pin are not possible in certain patients. Further modifications of nail design may be required to suit the Asian population. 


\section{REFERENCES}

1. Seinsheimer F. Subtrochanteric fractures of the femur. J Bone Joint Surg Am. 1978; 60: 300-6.

2. Craig NJ, Sivaji C, Maffulli N. Subtrochanteric fractures. A review of treatment options. Bull Hosp Jt Dis. 2001; 60:35-46.

3. Sims SH. Subtrochanteric femur fractures. Orthop Clin North Am. 2002; 33: 113-26.

4. Parker MJ, Dutta BK, Sivaji C, Pryor GA. Subtrochanteric fracture of the femur. Injury. 1997; 28(2): 91-5.

5. Kinast C, Bolhofner BR, Mast JW, Ganz R. Subtrochanteric fractures of the femur. Results of treatment with the 95 degrees condylar blade-plate. Clin Orthop Relat Res. 1989; 238: 122-30.

6. Waddell JP. Subtrochanteric fractures of the femur: a review of 130 patients. J Trauma. 1979; 19: 582-92.

7. Watson HK, Campbell RD Jr, Wade PA. Classification, treatment and complications of the adult subtrochanteric fracture. $J$ Trauma. 1964; 60: 457-80.

8. Curtis MJ, Jinnah RH, Wilson V, Cunningham BW. Proximal femoral fractures: a biomechanical study to compare intramedullary and extramedullary fixation. Injury. 1994; 25(2): 99-104.

9. Simmermacher RK, Bosch AM, Van der Werken C. The AO/ASIF-PFN (PFN): a new device for the treatment of unstable proximal femoral fractures. Injury. 1999; 30: 327-32.

10. WM Gadegone, YS Salphale. PFN - an analysis of 100 cases of proximal femoral fractures with an average follow up of 1 year. International Orthopaedics (SICOT). 2007; 31: 403-8.

11. BF Ongkiehong, R Leemans. PFN failure in a subtrochanteric fracture: The importance of fracture to distal locking screw distance. Injury Extra. 2007; 38: 445-50.

12. Ramakrishnan M, Prasad SS, Parkinson RW, Kaye JC. Management of subtrochanteric femoral fractures and metastases using long PFN. Injury. 2004; 35: 184-90.

13. Morihara T, Arai Y, Tokugawa S, Fujita S, Chatani K, Kubo T. PFN for treatment of trochanteric femoral fractures. Journal of Orthopaedic Surgery 2007; 15(3): 273-7.

14. Schipper IB, Steyerberg EW, Castelein RM, van der Heijden FH, den Hoed PT, Kerver AJ, et al. Treatment of unstable trochanteric fractures. Randomised comparison of the Gamma nail and the PFN. J Bone Joint Surg Br 2004; 86: 86-94.

15. Banan H, Al-Sabti A, Jimulia T, Hart AJ. The treatment of unstable, extracapsular hip fractures with the AO/ASIF PFN (PFN) our first 60 cases. Injury. 2002; 33: 401-5.

16. Kawaguchi S, Sawada K, Nabeta Y. Cutting-out of the lag screw after internal fixation with the Asiatic gamma nail. Injury. 1998; 29: 47-53.

17. Papasimos S, Koutsojannis C M, Panagopoulos A, Megas P, Lambiris E. A randomised comparison of AMBI, TGN and PFN for treatment of unstable trochantric fractures. Arch Orthop Trauma Surg. 2005; 125: 462-8.

18. Lei-Sheng Jiang, Lei Shen, Li-Yang Dai. Intramedullary fixation of subtrochantric fractures with long PFN or long gamma nail: Technical notes and preliminary results. Ann Acad Med Singapore. 2007; 36: 821-6. 